\title{
Sources of Solar Wind at Solar Minimum: Constraints from Composition Data
}

\author{
Thomas H. Zurbuchen • Rudolf von Steiger • \\ Jacob Gruesbeck • Enrico Landi • Susan T. Lepri • \\ Liang Zhao • Viggo Hansteen
}

Received: 9 November 2011 / Accepted: 2 April 2012 / Published online: 26 April 2012

(C) The Author(s) 2012. This article is published with open access at Springerlink.com

\begin{abstract}
In this discussion of observational constraints on the source regions and acceleration processes of solar wind, we will focus on the ionic composition of the solar wind and the distribution of charge states of heavy elements such as oxygen and iron. We first focus on the now well-known bi-modal nature of solar wind, which dominates the heliosphere at solar minimum: Compositionally cool solar wind from polar coronal holes overexpands, filling a much larger solid angle than the coronal holes on the Sun. We use a series of remote and in-situ characteristics to derive a global geometric expansion factor of $\sim 5$. Slower, streamer-associated wind is located near the heliospheric current sheet with a width of $10-20^{\circ}$, but in a well-defined band with a geometrically small transition width. We then compute charge states under the assumption of thermal electron distributions and temperature, velocity, and density profiles predicted by a recent solar wind model, and conclude that the solar wind originates from a hot source at around 1 million $\mathrm{K}$, characteristic of the closed corona.
\end{abstract}

Keywords Solar wind · Solar corona composition

T.H. Zurbuchen $(\bowtie) \cdot$ J. Gruesbeck · E. Landi · S.T. Lepri $~$ L. Zhao

Department of Atmospheric, Oceanic and Space Sciences, University of Michigan, Ann Arbor, MI 48109, USA

e-mail: thomasz@umich.edu

R. von Steiger

International Space Science Institute, Hallerstrasse 6, Bern, Switzerland

R. von Steiger

Physikalisches Institut, University of Bern, Sidlerstrasse 5, Bern, Switzerland

L. Zhao

NCAR/HAO, 3080 Center Green, Boulder, CO, USA

V. Hansteen

Institute of Theoretical Astrophysics, University of Oslo, P.O. Box 1053 Blindern, Oslo, Norway 


\section{Introduction}

During its first polar pass, Ulysses, a highly successful joint mission of the European Space Agency (ESA) and the National Aeronautics and Space Administration (NASA), revealed that there are important compositional differences between the two very different states of solar wind (Geiss et al. 1995). On the one hand, Ulysses observed fast solar wind at speeds of $\sim 800 \mathrm{~km} / \mathrm{s}$, which dominated the high-latitude heliosphere. This wind exhibited a composition indicating that it was born in relatively cool regions on the Sun, with ionic charge states indicative of source temperatures $\sim 800,000 \mathrm{~K}$ to $1 \mathrm{MK}$. The elemental composition of this wind was observed to be comparable to that of the photosphere. Furthermore, this type of solar wind exhibited very little temporal and spatial variation in both elemental and ionic charge state composition (von Steiger et al. 2000).

Near the heliospheric current sheet, on the other hand, the composition of the solar wind plasma was observed to be different from coronal hole-associated fast wind. This slower wind, with speeds $\sim 400 \mathrm{~km} / \mathrm{s}$, exhibited ionic charge states that reflected source temperatures in excess of $1.2 \mathrm{MK}$, significantly hotter than those of the coronal hole-associated wind. Its elemental abundances were observed to be significantly biased as compared to photospheric abundances. In particular, elements with first ionization potential (FIP) $<10 \mathrm{eV}$ (i.e., metals such as $\mathrm{Fe}, \mathrm{Mg}$, etc.) were observed to be enhanced relative to $\mathrm{O}$ and elements with larger FIP. The variability of all compositional quantities was also observed to be much larger than in coronal hole-associated fast wind (Zurbuchen and von Steiger 2006; von Steiger et al. 2010).

These observations continued until the end of the Ulysses mission (Wenzel et al. 1992) in mid-2009, providing a new and unprecedented view of the three-dimensional heliosphere, its solar and interstellar boundaries and their sources, and the particle populations that shape the solar wind plasma and fields. Ulysses provided data for almost 19 years, covering nearly an entire solar cycle. It covered a heliocentric range of 1 to $5.4 \mathrm{AU}$, and nearly three orbits, each of which lasted approximately 6.2 years. Also, due to Ulysses' high inclination of $80.2^{\circ}$, its observations spanned all solar magnetic latitudes. During its entire orbit, Ulysses SWICS continued to take measurements that confirmed the key observational results of the Geiss et al. (1995) paper discussed above, and tested their basic validity throughout the solar cycle (i.e., Zurbuchen et al. 2002). A representative data set is provided in Fig. 1. Clearly, the ordering of ionization temperature and elemental abundance is robust over nearly an entire solar cycle. Here, we use the $\mathrm{C}^{6+} / \mathrm{C}^{5+}$ ratio to remain consistent with previously published values, but notice that we recently identified $\mathrm{C}^{6+} / \mathrm{C}^{4+}$ as a very suitable temperature proxy for favorable statistical properties and because of the fact that both ionic charge states freeze in at approximately the same location near the Sun (Landi et al. 2012). Throughout this paper, we are using the average charge state of carbon. Details of the measurement techniques can be found in Gloeckler et al. (1992).

Another mission, the Solar and Heliospheric Observatory (SOHO) (Domingo et al. 1995), has simultaneously revolutionized our understanding of the processes that heat the corona and shape the solar wind. One of SOHO's key observations suggested a similar dichotomy of solar plasma compositions in the closed corona as had been observed in situ (Feldman and Widing 1999; Feldman et al. 1999): cooler (<0.8 MK) loops within coronal holes exhibit nearly photospheric elemental composition, qualitatively consistent with that observed in fast wind at high latitude. In contrast, the closed corona at electron temperatures 1.4 MK exhibited elemental fractionation, in qualitative and approximately quantitative agreement with slow streamer wind.

These combined observations provide important constraints for models of the solar wind and corona, but result in a theoretical challenge that is addressed elsewhere (i.e., Fisk and 


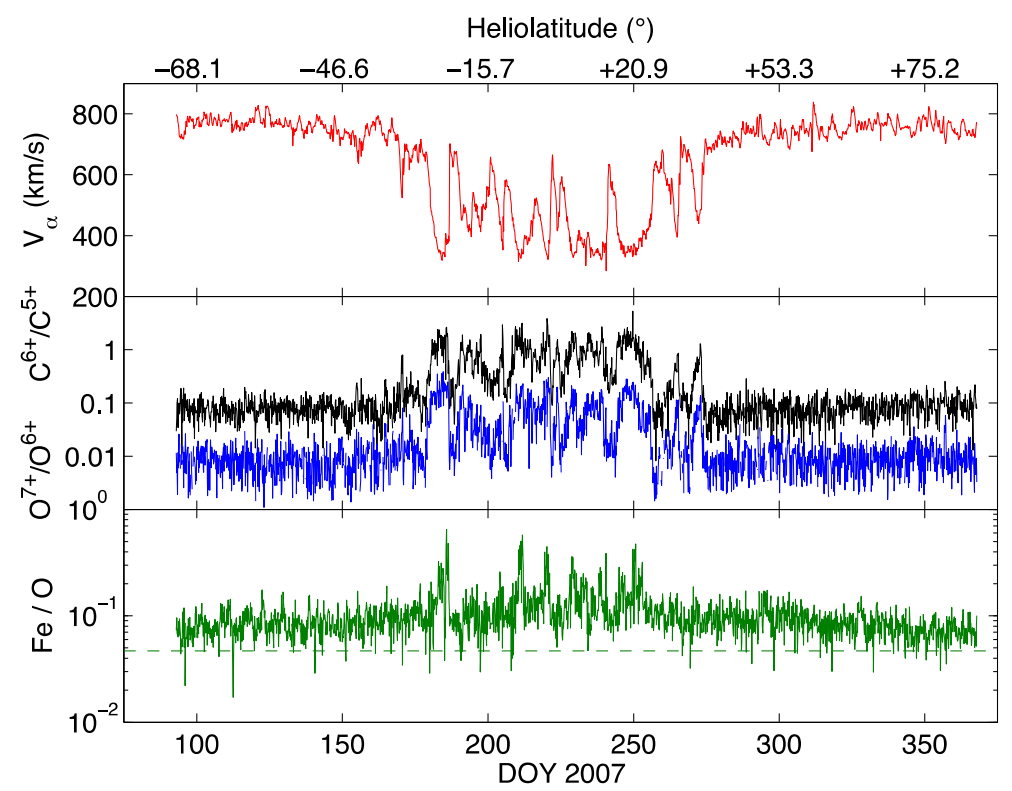

Fig. 1 Ulysses SWICS data during the fast latitude scan before mission termination. The panels show the speed of solar wind alpha particles, the ratio of $\mathrm{O}^{7+} / \mathrm{O}^{6+}$, and $\mathrm{C}^{6+} / \mathrm{C}^{5+}$, as well as the elemental ratio of $\mathrm{Fe} / \mathrm{O}$. The average photospheric $\mathrm{Fe} / \mathrm{O}$ value is shown as a dashed line. Also indicated on the plot is the heliographic latitude. Coronal hole-associated wind is cooler, indicated by smaller ionic abundance ratios, and also more photosphere-like with respect to its elemental composition

Zurbuchen 2006; Zurbuchen 2007; Antiochos et al. 2011a): How does plasma from the magnetically closed corona escape to form a magnetically open heliosphere? By whatever method, plasma from the quiet closed corona is apparently heated, accelerated, and released to fill a significant fraction of the heliosphere (Feldman and Widing 1999; Feldman et al. 1999).

This analysis will focus on the compositional properties of the solar wind and look at two key quantities that provide important constraints for solar wind models-the spatial distribution of coronal hole wind and streamer wind, and the heating and acceleration processes of the solar wind. Both investigations heavily rely upon the compositional results described previously, but use a set of assumptions and models to derive constraints that should be valid for a broad set of models for the solar wind.

\section{Spatial Extension of Streamer-Associated Slow Wind}

Due to the observed variability in ionic and elemental composition in the solar wind, these quantities are ideally suited, in principle, to track solar wind to its coronal source (von Steiger et al. 2000; Zurbuchen et al. 2002). However, such comparisons are complicated somewhat by the fact that they rely on a series of observational quantities all of which have some limitations (such as field-of-view integrations, low statistics in some key lines, or lack of coverage) and therefore a need for interpretation. Thus, such comparisons are complicated by the fact that they need to be carried out using remote and in-situ observations of the same plasma parcel. This can only be done when remote-sensing and in-situ instruments 
are in quadrature at time-stationary conditions. Such a configuration, however, can only be achieved on a limited number of occasions. Even during quadrature, line-of-sight integration of remote-sensing data can be complicated when multiple plasmas are present along the line of sight (e.g. Hahn et al. 2011).

Here we use three types of measurements to define the boundary between coronal holeassociated solar wind and streamer-associated wind, as shown in Fig. 2. First, we use images of the Sun at 195 A made by the Extreme-ultraviolet Imaging Telescope (EIT) (Delaboudinière et al. 1995). Coronal holes in these images are identified by dark regions due to their lower electron temperature, which leads to reduced extreme-ultraviolet (EUV) emissions in these regions. Representative images are provided in the top of Fig. 2. Gradients at the limb are indicated by a hash mark. They are found at around $60^{\circ}$ heliographic latitude, with some variability of about $10^{\circ}$ due to geometric and temporal effects.

During its expansion into the inner heliosphere, the solid angle filled by fast wind from coronal holes increases as it moves away from the Sun. This angular expansion is observed by the SOHO UltraViolet Coronagraph Spectrometer (UVCS) (Kohl et al. 1998), which provides synoptic observations of hydrogen Ly-alpha and OVI, here provided at three different radial distances (Fig. 2, middle). Clear gradients are visible as the darker area, signifying coronal holes, expands to fill more space at larger radii, providing a clue about the expansion of the field from coronal holes to low latitudes. Again, these gradients can be used to identify the interface regions between fast and slow wind.

Finally, the in-situ location of the boundary between solar wind coming from coronal holes and the streamer belt can be determined using the Ulysses SWICS measurements discussed previously. Since the direct one-to-one analysis of Ulysses data with spectroscopic data is not possible because SWICS only observes at one location at a given time, a whole set of boundary crossings are given for the time period from June 1996 to January 1997. The variability shown is more a measure of the large-scale geometric changes of the stream interface than an uncertainty of its determination. In fact, the determination of the location of the stream interface can be done very accurately, as shown by Wimmer-Schweingruber et al. (1997), because of the steepness of the gradient between the two compositional regimes.

The key results of the three measurement types described above and in Fig. 2 can be combined and approximated by a simple expansion law, as shown in Fig. 3. The observations imply an expansion ratio of $E \sim 5$, as is easily computed under the assumption of cylindrical symmetry and assuming that the latitudinal expansion of coronal holes is to latitude $\theta_{C H}$ and the expansion in the solar wind is to latitude $\theta_{S W}$.

$$
E=\frac{1-\sin \left(\theta_{S W}\right)}{\theta_{C H}} .
$$

Such expansion factors have been analyzed on the basis of magnetic field calculations and are in qualitative agreement (Wang et al. 1997). Referring to such an analysis, Wang et al. also argue that this expansion process would lead to transient reconnection processes with closed magnetic field at the streamer edges. In fact, the plasma released should have intermittent reconnection, observed as "blobs" when using line-of-sight integrating coronagraph images (Sheeley et al. 1997). In fact, the top of streamers may be intrinsically unstable, naturally resulting in such intermittent ejections (Chen et al. 2009).

However, there is a critical aspect of Fig. 3, further emphasized in Fig. 4, which uses the analysis methodology described by Zhao et al. (2009) and combines SWICS data from Ulysses and the Advanced Composition Explorer (ACE) from 1990 to 2010 and 1998 to 2010, respectively. For each Carrington rotation, the measured solar wind $\mathrm{O}^{7+} / \mathrm{O}^{6+}$ ratio is mapped into a magnetic coordinate system, effectively calculating the normal distance 


\section{July 1996}

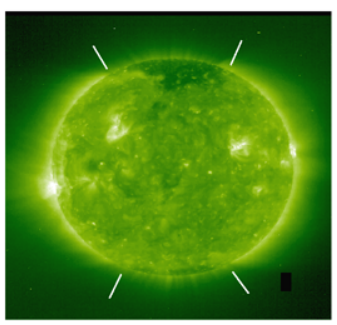

1 Jan 1997

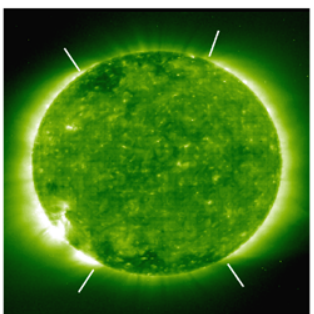

30 June 1997

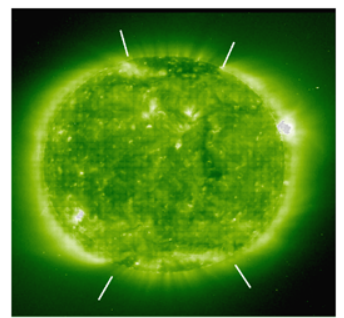

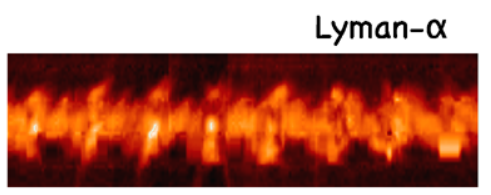

\section{OVI}
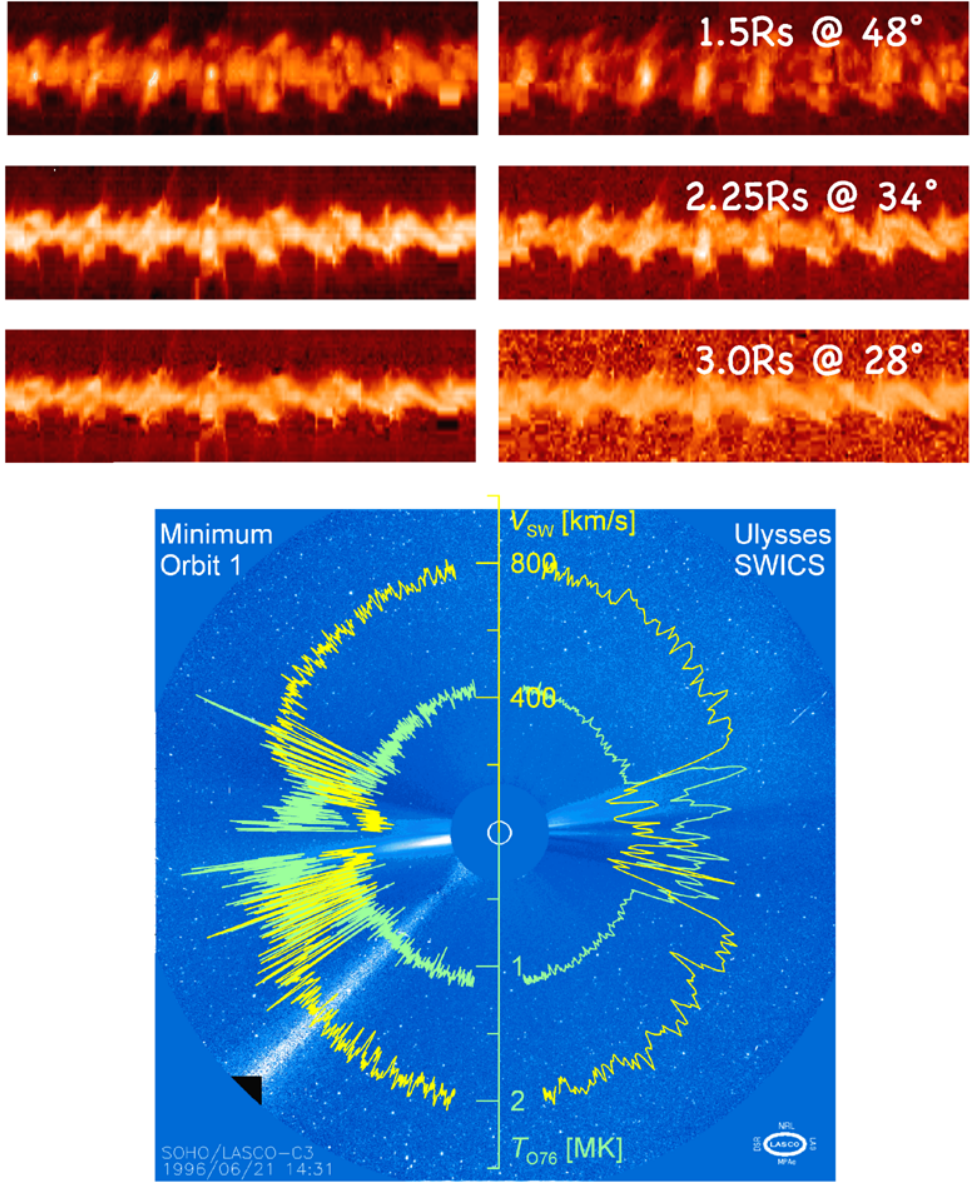

Fig. 2 Solar remote and in-situ observations tracking the boundary between fast and slow wind to the Sun. The observations constraining coronal holes near the Sun use images from SOHO-EIT at $19.5 \mathrm{~nm}$. Coronal hole boundaries are indicated with a hash mark based on brightness gradients in this line. The near-coronal expansion of coronal hole plasmas is constrained by contemporary synoptic scans of Lyman-alpha and OVI over Carrington rotations 1910.85 to 1918.69. Coronal hole boundaries are defined again based on brightness gradients. Heliospheric boundaries are defined based on coronal temperatures, which are calculated from $\mathrm{O}^{7+} / \mathrm{O}^{6+}$, under the assumption of local thermal equilibrium 
Fig. 3 Boundaries derived from solar EUV observations by SOHO-EIT, synoptic scans from SOHO-UVCS at various heliocentric distances and Ulysses-SWICS as shown in Fig. 2 and discussed in the text. On average, the magnetic field rooted in coronal holes at latitudes of $60^{\circ}$ or less expands over a latitude range of $\sim 40^{\circ}$

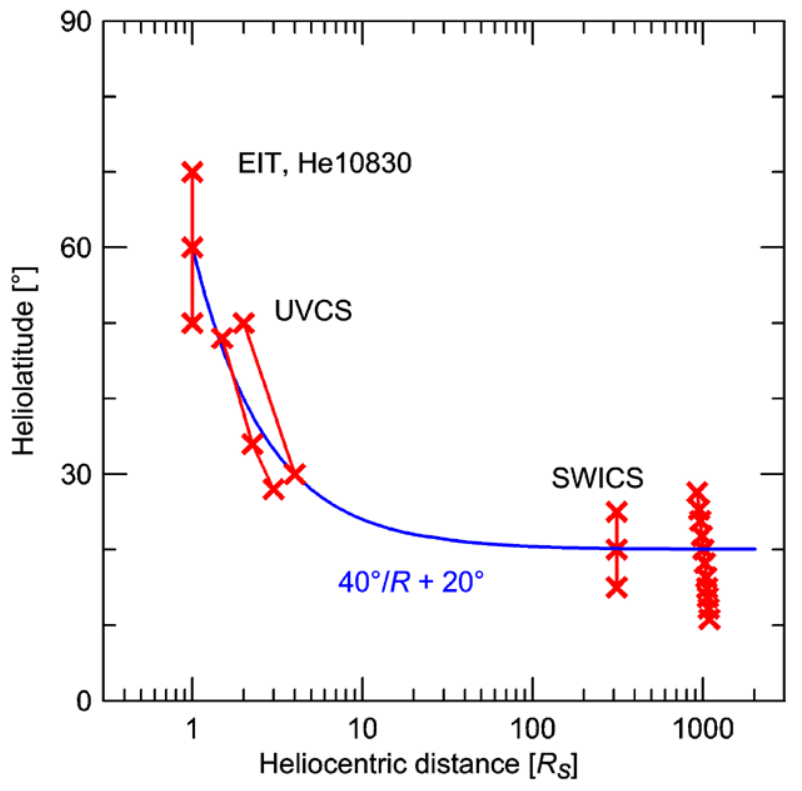

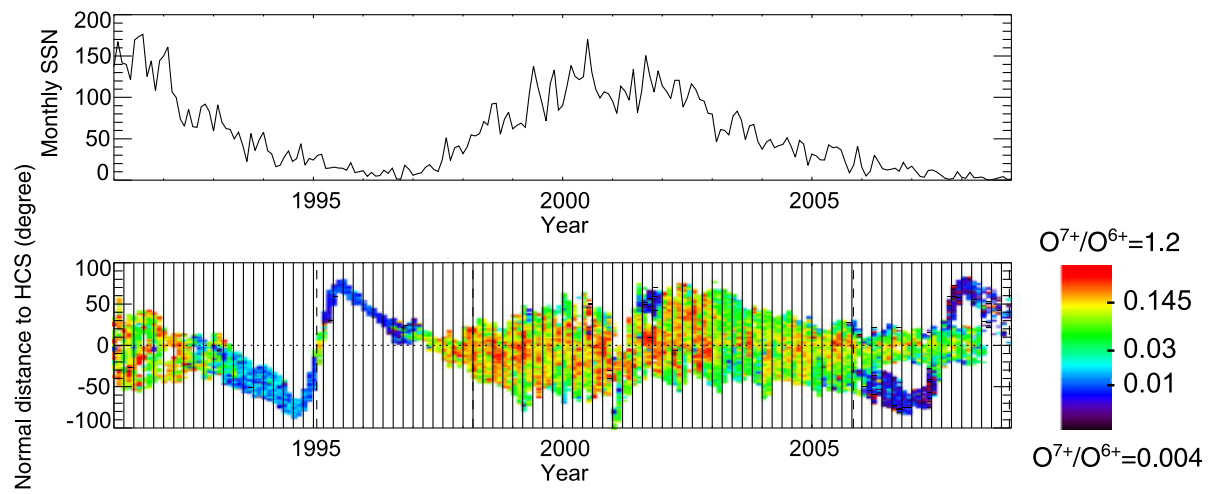

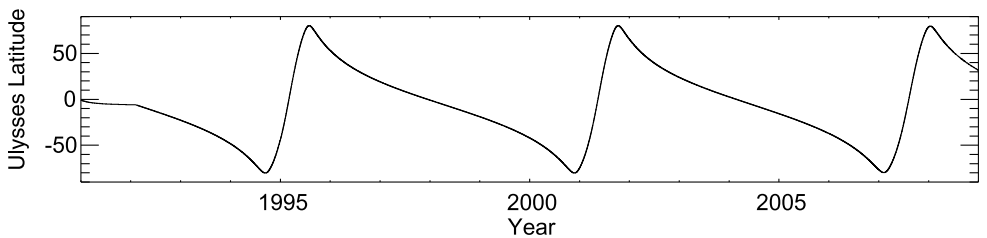

Fig. 4 Solar wind $\mathrm{O}^{7+} / \mathrm{O}^{6+}$ measurements from Ulysses and ACE mapped into a magnetic coordinate system. Slow wind and also coronal mass ejections at elevated $\mathrm{O}^{7+} / \mathrm{O}^{6+}$ ratios are observed within $20^{\circ}$ of the current sheet near the solar minimum. This band can expand as high as $80^{\circ}$ in latitude during solar maximum

from the footpoints of the solar wind on a 2.5 solar radii surface to the current sheet (see Zhao et al. 2009 and Zhao and Fisk 2011 for details). Thus, geometric variations along the $y$-axis in the middle panel of Fig. 4 include both orbit variations and geometric changes of 
the heliospheric current sheet. Oxygen composition data, $\mathrm{O}^{7+} / \mathrm{O}^{6+}$, are then averaged in $5^{\circ}$ intervals and color-coded.

Slow, streamer-associated wind-here identified by an $\mathrm{O}^{7+} / \mathrm{O}^{6+}>0.145$ (Zhao et al. 2009) - fills a significant part of the heliosphere. Under all circumstances, slow wind dominates magnetic latitudes at $20^{\circ}$ or lower, consistent with the analysis in Fig. 3, or $35 \%$ of the solid angle in the heliosphere, under the assumption of a spherical ring with this $20^{\circ}$ half-width. It is therefore very difficult to imagine that it might originate from a transient boundary process such as the one proposed by Wang et al. (1997) or others. Slow wind is a geometrically extended phenomenon, filling a substantial fraction of the heliosphere at all times (i.e., Schwenn 1990). This comparatively large extension is also the principal reason why slow wind is such an important contributor to the Earth's space environment (Zhao et al. 2009; Zhao and Fisk 2011). If slow wind only occurred within $\sim 1^{\circ}$ of the streamer, perhaps associated with the dynamic evolution of the streamer stalk, we would not likely find fractionated slow wind near Earth for over $30 \%$ of the time under most conditions (see also Schwenn 2007).

\section{Solar Wind Heating from Ionic Charge States}

It is possible to utilize ionic charge states to constrain the physical properties that lead to heating of solar wind plasma near the Sun and thus the acceleration of the solar wind. Such constraints can be developed in a qualitative sense (i.e., Geiss et al. 1995; or von Steiger and Zurbuchen 2011), or they can be done quantitatively (Ko et al. 1997; Laming and Lepri 2007; Gruesbeck et al. 2011).

Landi et al. (2012) recently discussed the challenges of qualitative measures, the socalled freeze-in temperatures, as a diagnostic for electron temperatures in the low corona. Although it is possible to calculate freeze-in temperatures from any charge-state ratio of a given element under the assumption of thermal equilibrium, these freeze-in temperatures are substantially different from the local temperatures near the freeze-in point. This is caused by non-local effects dominating the freeze-in process (i.e., the ionic charge distribution at a given near-solar distance $r_{0}$ is dependent on the thermal properties over a large range of distances $r<r_{0}$ ), especially when the ionization and recombination scales of two ions in a given ion ratio exhibit differences (Landi et al. 2012). Such non-local effects were shown to be particularly important for $\mathrm{O}^{7+} / \mathrm{O}^{6+}$ ratios, but they are less important for $\mathrm{C}^{6+} / \mathrm{C}^{4+}$. Thus, specific conclusions about source temperatures of solar wind source regions, such as by Schwadron and McComas (2003), likely are affected by these non-local effects.

Frozen-in ion charge state distributions can be calculated for a given set of ionization and recombination rates (Mazzotta et al. 1998) by solving a continuity equation for each ionic charge state of a given element (see Gruesbeck et al. 2011) for a given combination of radial profiles of velocity, density, and electron temperatures. Ion temperatures within reasonable and observed ranges (i.e., by Kohl et al. 1998) have negligible impact on the freeze-in process. Here we use such a calculation to point out that there is generally a rather significant difference between our observed ionic charge distributions and the ones predicted by model calculations.

Among the currently used solar wind heating and acceleration models, we choose the one by Hansteen (see Hansteen and Leer 1995; Leer et al. 1998) to illustrate a key result we also found similarly for other models. This particular model covers the region from the middle chromosphere through the transition region and corona out into the solar wind, and solves the continuity, momentum, and energy equations for various species of hydrogen and 
Fig. 5 Solar wind density, velocity, and electron temperature for fast and slow wind as computed by Hansteen's model described in the text. Fast wind profiles are shown in blue and slow wind profiles in red. These profiles are used to calculate frozen-in charge state distributions for comparison with Ulysses data
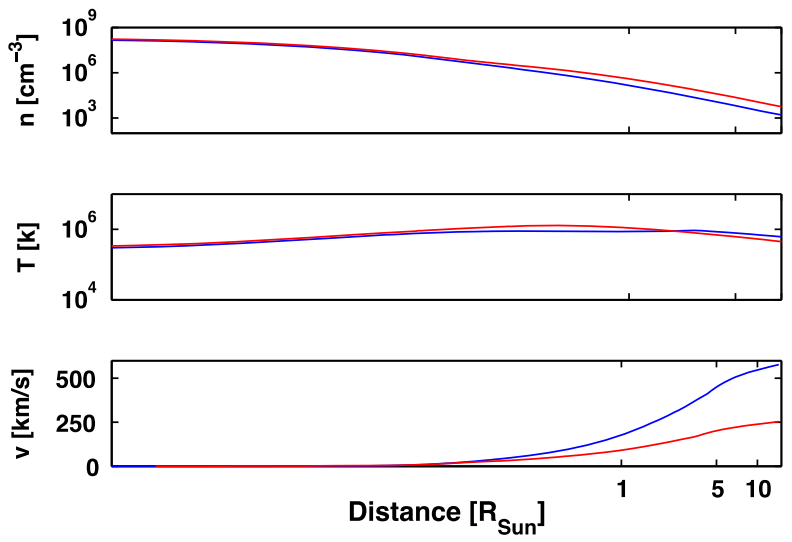

helium as well as electrons. A parameterized "mechanical" heating flux of $100 \mathrm{~W} / \mathrm{m}^{2}$ is inserted in the corona in both models: the energy flux is spread through thermal conduction and through the acceleration of the solar wind. In the fast wind the mechanical energy flux is deposited at 2.5 solar radii (Rs), of which $66 \%$ goes into protons, $26 \%$ into alpha particles, and the remaining $8 \%$ into electrons. Energy is deposited much lower in the slow wind model - at 0.2 Rs, with $45 \%$ going into protons, $35 \%$ into alpha particles, and the remaining $20 \%$ into electrons. The scale height of the heating is in both cases set to 0.2 Rs. Figure 5 shows velocity, density, and electron temperature profiles for both fast and slow solar wind. The overall properties of the computed solar wind streams are in good agreement with the observations from Ulysses.

The profiles in electron density, temperature, and solar wind velocity in Fig. 5 are now used to compute frozen-in charge state distributions and to compare them with SWICS data measured between 1.34 and 2 AU during fast latitude scans in 1995, 2001, and 2007, as shown in Fig. 1. These fast scans have durations of approximately 100 days and include coronal hole-associated fast wind as well as slow wind from streamers. During this time, Ulysses crossed the latitude range from $-80^{\circ}$ to $80^{\circ}$. There are also some contributions from CME-associated plasmas, with elevated ionic charge states as compared to slow wind (see references within Zurbuchen and Richardson 2006). However, these contributions from CMEs were very rare during the three fast scans of Ulysses, as can be seen from the small number of isolated data points at high charge state temperatures in Fig. 9.

Figures 6 and 7 show the freeze-in process for heavy ions in fast and slow wind, respectively. In both cases, for simplicity, it was assumed that the heavy ions propagate at the speed of protons. Observations between 0.3 and 1 AU suggest differential speeds between alpha particles and protons of the order of the local Alfvén speed (Schwenn 1990; Esser and Edgar 2001). We currently do not have any good observational constraints for such differential speeds of heavy ions ( $m>4 \mathrm{amu}$ ), except near $1 \mathrm{AU}$ and up to $5.6 \mathrm{AU}$, where heavy ions are observed to propagate at rather comparable speeds to alpha particles (von Steiger and Zurbuchen 2006).

Figure 8 compares the frozen-in ionic distributions for fast wind streams to the ionic observations from Ulysses. Here, we use a time resolution of 2 hours, which provides sufficient statistics for all measurements. A simple criterion based on $\mathrm{C}$ and $\mathrm{O}$ charge states was used to separate coronal hole-associated wind from wind associated with streamers (von Steiger et al. 2010). Figure 8a indicates the speed and $\log \left(\mathrm{O}^{7+} / \mathrm{O}^{6+}\right)$ distribution. Although most coronal hole-associated wind during Ulysses' fast latitude scans is observed at speeds between 700 and $900 \mathrm{~km} / \mathrm{s}$, there are some cool, coronal hole-associated wind streams at rather 


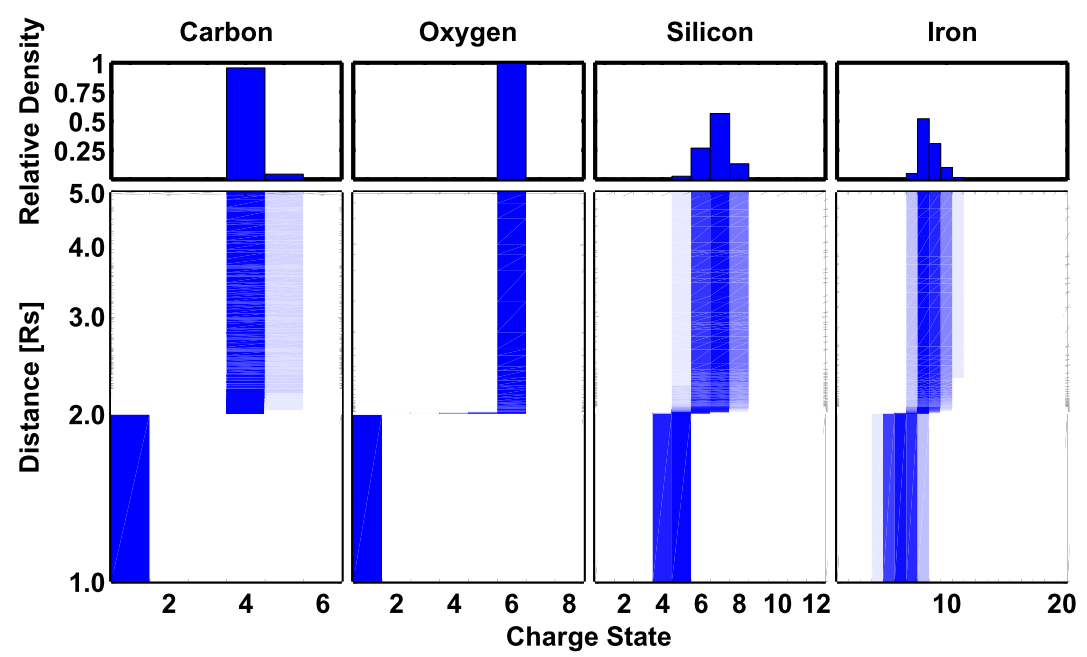

Fig. 6 Freeze-in process of $\mathrm{C}, \mathrm{O}, \mathrm{Si}$, and $\mathrm{Fe}$ in fast solar wind, as described in Fig. 5. The profiles show the radial evolution and freeze-in near the Sun. The freeze-in process is completed within 3 solar radii. The change of ionic charge states near the temperature maxima occurs very quickly and is not discernible within the resolution of this plot

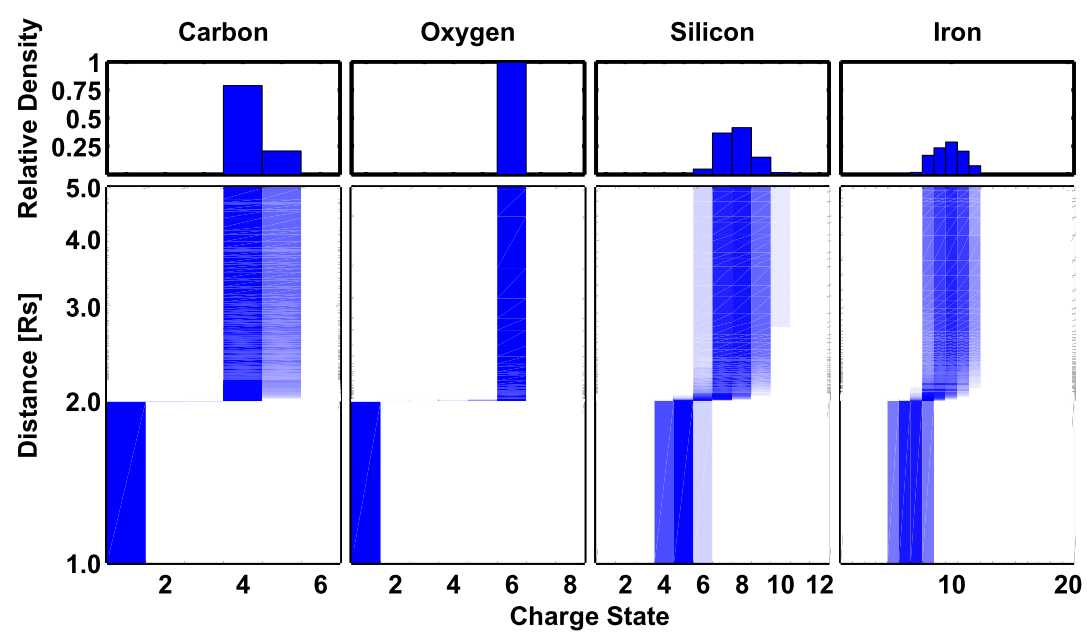

Fig. 7 Freeze-in process of $\mathrm{C}, \mathrm{O}, \mathrm{Si}$, and Fe in slow wind, as described in Fig. 5

low speed. Thus, clearly, the solar wind speed is not an ideal indicator for the origin of solar wind. Also, note that the range of $\log \left(\mathrm{O}^{7+} / \mathrm{O}^{6+}\right)$ observed values is extended over a larger rate than described by Geiss et al. (1995), for example. This enhanced range is caused by a systematic cooling of the corona during the past 20 years, as described in von Steiger and Zurbuchen (2011), which leads to a broadening of the ionic charge distribution. In fact, the coronal hole solar wind observed during the last Ulysses orbit, and shown in Fig. 1, exhibits the coolest ionic charge states observed during the entire Ulysses mission. 

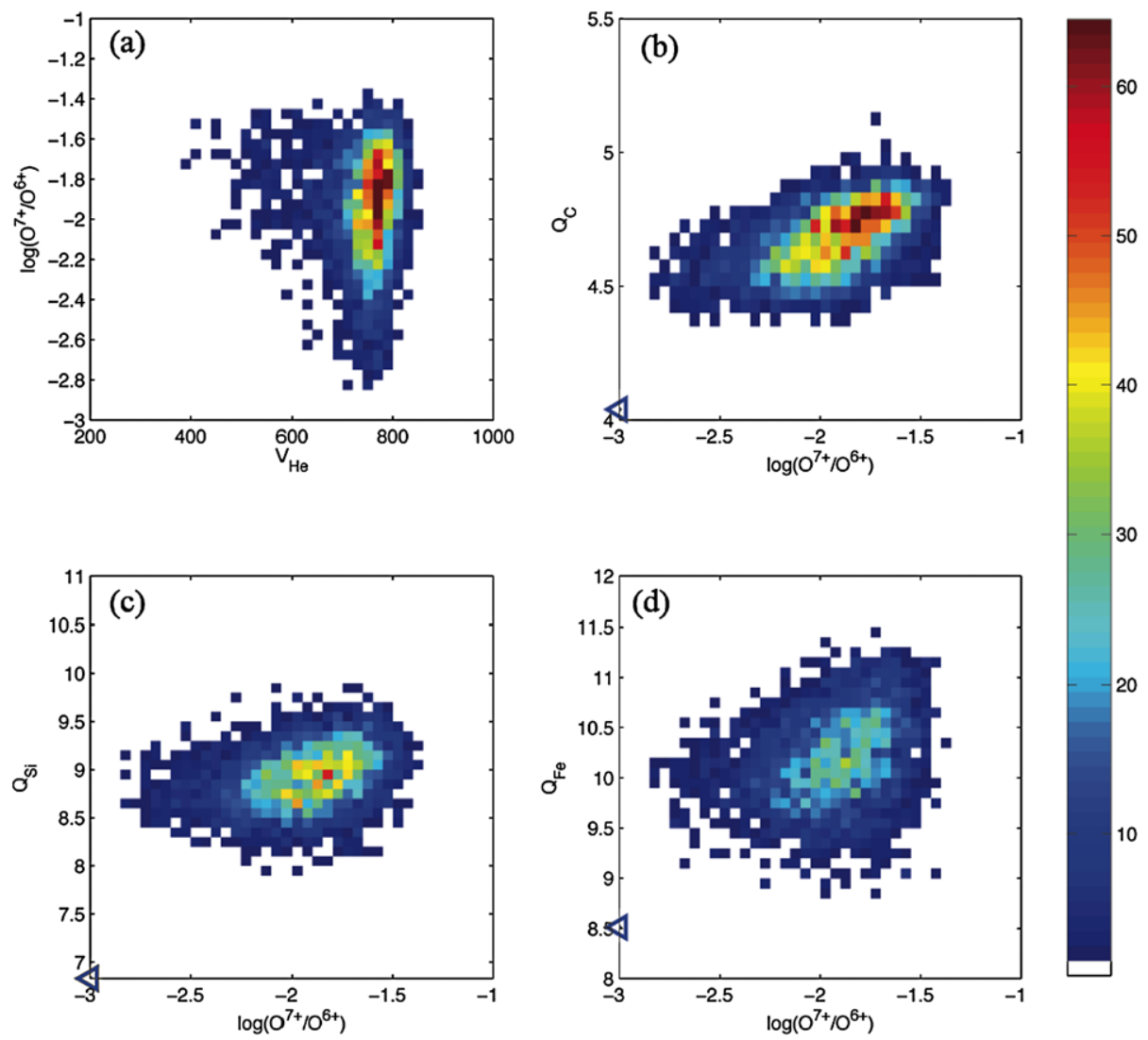

Fig. 8 Ionic charge properties of coronal hole-associated wind and their comparison with Hansteen's model prediction: (a) shows measured $\log \left(\mathrm{O}^{7+} / \mathrm{O}^{6+}\right)$ as a function of helium velocity, (b)-(d) show the measured average carbon, silicon, and iron charge states as a function of $\log \left(\mathrm{O}^{7+} / \mathrm{O}^{6+}\right)$. The model, shown as a triangular symbol, underestimates all charge states, resulting in distributions that are consistently too cool. This difference is the most extreme for $\mathrm{C}$ and $\mathrm{O}$ charge states, which freeze in very close to the Sun

Figure $8 \mathrm{~b}$ shows the relation of the average charge state of $\mathrm{C}$ with $\log \left(\mathrm{O}^{7+} / \mathrm{O}^{6+}\right)$. Both ionic charge states are expected to freeze in rather close to the Sun (see, e.g., von Steiger and Zurbuchen 2011). Also, it is a well-known observational fact that ionic charge states of $\mathrm{C}$ and $\mathrm{O}$ correlate over a large range of solar wind conditions (von Steiger 2008). This correlation can again be found here, although the distribution is broadened by the admixture of plasma components of significantly different coronal hole temperatures. The correlation is strong over a large part of the $\log \left(\mathrm{O}^{7+} / \mathrm{O}^{6+}\right)$ range, but the average $\mathrm{C}$ charge state does not seem to decrease over $\log \left(\mathrm{O}^{7+} / \mathrm{O}^{6+}\right)<-2$, indicating a statistical limitation of the $\mathrm{O}^{7+} / \mathrm{O}^{6+}$ measurements at the 3-hour time resolution used here-because of the very low ratio, there are almost no $\mathrm{O}^{7+}$ measurements in a given time interval. The blue triangle in the bottom right part of the plot shows the result computed from Fig. 6. The solar wind model parameters are significantly smaller than the actual fast wind data. For example, if the modeled $\log \left(\mathrm{O}^{7+} / \mathrm{O}^{6+}\right)$ was translated into freeze-in temperature, it would show that $T_{f}=0.65 \mathrm{MK}$ and not the temperatures around $T_{f}=1.1 \mathrm{MK}$ found in the data. The electron temperature of the model, shown in Fig. 5, is obviously significantly higher. Yet, because 

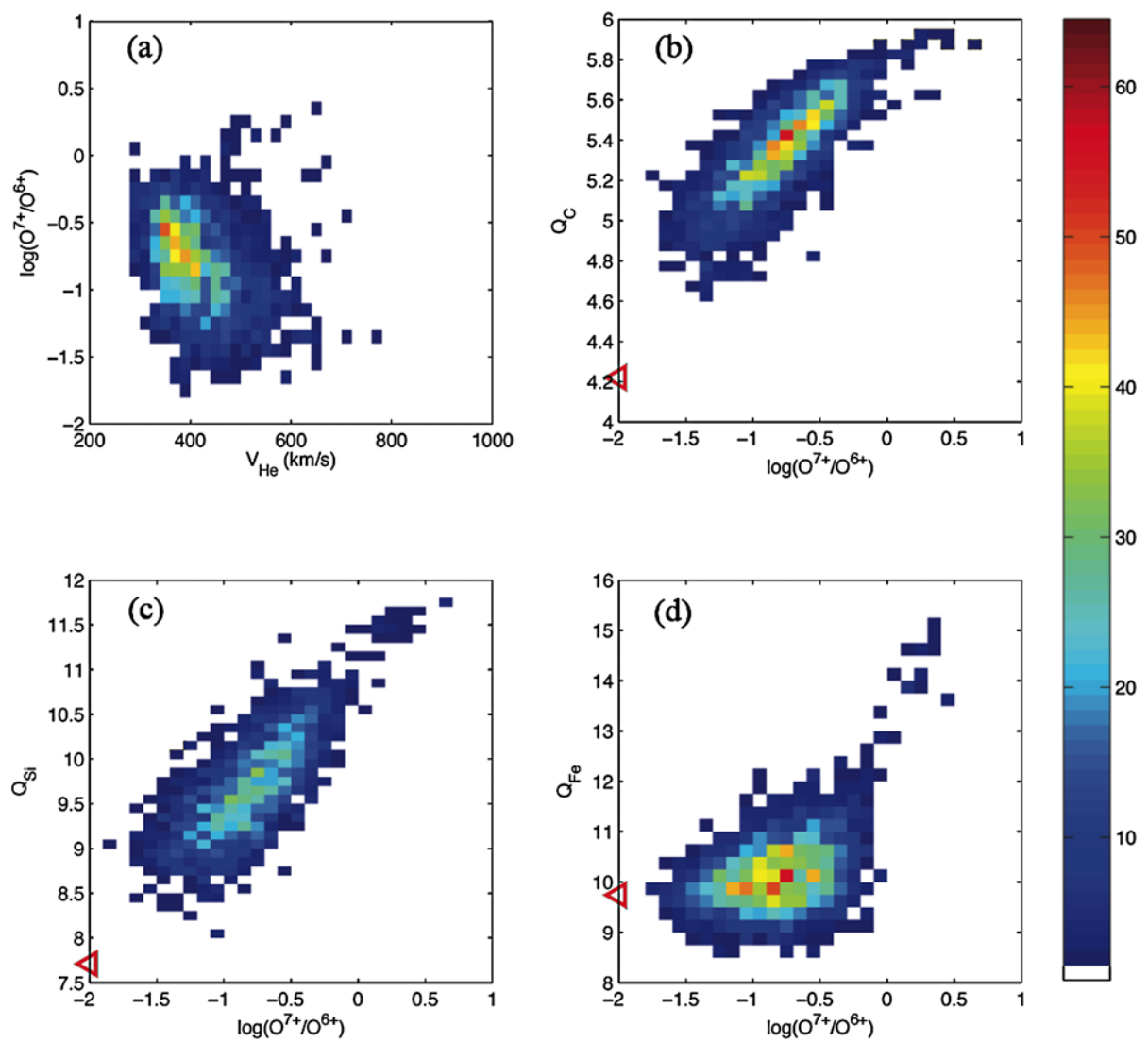

Fig. 9 Ionic charge properties of streamer-associated wind and their comparison with the Hansteen's model predictions, in the same format as Fig. 8. The model underestimates charge states that freeze in close to the Sun, such as $\mathrm{C}$ and $\mathrm{O}$. Si is also underestimated. However, the Fe prediction is in the same range as the observations

of the already significant speed, the charge state evolution is too slow to adapt to these higher temperatures. We have done similar calculations with a number of solar wind models, such as Cranmer et al. (2007) and van der Holst et al. (2010), and have found qualitative consistency with the models: a coronal hole solar wind that produces an in-situ solar wind with dynamic properties consistent with observations tends to underestimate significantly the ionic charge states of $\mathrm{C}$ and $\mathrm{O}$.

Figures $8 \mathrm{c}$ and $8 \mathrm{~d}$ relate the $\log \left(\mathrm{O}^{7+} / \mathrm{O}^{6+}\right)$ to the average charge states of $\mathrm{Si}$ and $\mathrm{Fe}$, respectively. $\mathrm{Si}$ and $\mathrm{Fe}$ freeze in much later in the solar atmosphere, perhaps around 1.5-2 Rs (see von Steiger and Zurbuchen 2011). Due to their multitude of charge states, this freeze-in is very much non-local. Both $\mathrm{Si}$ and $\mathrm{Fe}$ charge states correlate with $\log \left(\mathrm{O}^{7+} / \mathrm{O}^{6+}\right)$, indicating a strong correlation of freeze-in conditions in the near solar corona with those in the far corona. This correlation is again less distinct at $\log \left(\mathrm{O}^{7+} / \mathrm{O}^{6+}\right)<-2$, again suggesting a statistical limitation of the $\mathrm{O}^{7+} / \mathrm{O}^{6+}$ ratio, as discussed by Landi et al. (2012). In both $\mathrm{Si}$ and Fe, the model calculations underestimate the measured values. If expressed as a proxy for the equivalent freeze-in temperature, the modeled $Q_{\mathrm{Si}}$ is equivalent to a freeze-in temperature of $T_{f}=0.8 \mathrm{MK}$ as compared to the measured range of 1.3-1.4 MK, and the modeled 
$Q_{\mathrm{Fe}}$ is at $T_{f}=1.0 \mathrm{MK}$ as compared to the measured $1.2-1.3 \mathrm{MK}$ range discussed in von Steiger and Zurbuchen (2011).

Figure 9 shows the result of the comparison between the model predictions and observations of streamer wind identified using ionic charge states (see von Steiger et al. 2010 for details). When comparing Fig. 8 to Fig. 9, the significantly larger ranges of all ionic quantities is evident. In general, streamer wind has a much larger variability than coronal hole solar wind in almost all dynamic and compositional signatures. This is one of the defining characteristics of slow wind (Gosling 1997; Zurbuchen and von Steiger 2006) and has been interpreted as a signature of a multitude of sources for this wind with large variability between various source regions.

Figure 9a first relates the speed of solar wind He, which is that of solar wind protons to within a few percent, to the $\log \left(\mathrm{O}^{7+} / \mathrm{O}^{6+}\right)$ values. Most measured speeds are around $400 \mathrm{~km} / \mathrm{s}$, but the range extends to higher speeds, mostly caused by the addition of plasmas from coronal mass ejections, but also because streamer wind can in fact be found at higher speeds. The correlation between $\log \left(\mathrm{O}^{7+} / \mathrm{O}^{6+}\right)$ and solar wind speed has been reported by Gloeckler et al. (2003), using the entire range of solar wind measurements. Figure $9 \mathrm{~b}$ relates the average $\mathrm{C}$ charge state to $\log \left(\mathrm{O}^{7+} / \mathrm{O}^{6+}\right)$ and compares it to the computed values from Hansteen's slow wind model shown in Fig. 5. Again, there is a strong correlation between $\log \left(\mathrm{O}^{7+} / \mathrm{O}^{6+}\right)$ and the average $\mathrm{C}$ charge state as they both freeze-in near the Sun. The model prediction is again significantly different from the observed values. Under local thermal equilibrium conditions, the modeled $\log \left(\mathrm{O}^{7+} / \mathrm{O}^{6+}\right)$ and average $\mathrm{C}$ charge state relates to $T_{f}=0.85 \mathrm{MK}$ and $T_{f}=0.71 \mathrm{MK}$, respectively. This should be compared to the range of values of $T_{f}=1.3-1.7 \mathrm{MK}$ derived from data (von Steiger 2008).

There is a very strong correlation in Fig. 9c between the average charge state of Si and the $\log \left(\mathrm{O}^{7+} / \mathrm{O}^{6+}\right)$ value. This correlation is significant and of similar quality as the one between $\mathrm{C}$ and $\log \left(\mathrm{O}^{7+} / \mathrm{O}^{6+}\right)$ shown in Fig. 9b. Such a close relationship is especially surprising considering the fact that $\mathrm{Si}$ and $\mathrm{O}$ freeze in at very different distances, perhaps more than $1.5 \mathrm{Rs}$ apart. Coronal mass ejections (CME) are well known to exhibit large log $\left(\mathrm{O}^{7+} / \mathrm{O}^{6+}\right)$ values and $\mathrm{Si}$ charge states are also enhanced (Zurbuchen and Richardson 2006; Gruesbeck et al. 2011). In Fig. 9c, data points with $\log \left(\mathrm{O}^{7+} / \mathrm{O}^{6+}\right)>0$ are $\mathrm{CME}$ associated and clearly discernible. Model predictions are again too low for the Si values-by almost 3 charge states from the center of the distribution. Assuming thermal equilibrium, the model would equate to $T_{f}=1.07 \mathrm{MK}$. The Fe distribution in Fig. 9d shows two distinct populationsone is streamer wind and the other is CME plasma, with a very strong contribution from the He-like ionic charge state of $\mathrm{Fe}^{16+}$ (Lepri et al. 2001). Gruesbeck et al. (2011) recently explained these high charge states as a result of rapid heating of high-density plasma with subsequent rapid cooling. The model prediction of $\mathrm{Fe}$ is consistent with the data, at freeze-in temperatures $T_{f}=1.1 \mathrm{MK}$, assuming equilibrium conditions.

\section{Discussion}

This analysis has two important conclusions. First, we analyzed the expansion properties of coronal hole-associated wind and inferred the latitudinal extent of solar wind from streamers. We conclude from this analysis that the closed corona is a large $(\sim 30 \%)$ contributor to the plasma of the solar wind. The release process of the slow wind is therefore not likely to be intermittent along coronal hole boundaries, as suggested by Wang et al. (1997). Instead, plasma comes from a larger area on the sun, as suggested by Fisk and Zurbuchen (2006) or Antiochos et al. (2011b). For a model to successfully explain our data, fractionated solar 
wind needs to be released into the heliosphere over a sufficiently large latitudinal range, up to $25^{\circ}$ from the current sheet.

It is possible that the streamer tip contributes to the heliospheric solar wind. In fact, Borrini et al. (1981) found a substantial difference of the helium abundance in the immediate vicinity of the heliospheric current sheet. The compositional signature only expanded from the current sheet for a rather short time. The time-resolution of heavy ions from SWICS is limited to $>1$ hour, so a very short signal may not be easily discernable.

We also address the important requirement for solar wind models to simultaneously predict the kinetic properties of the solar wind, as well as the ionic charge states. Typically, these models use higher-order-moments fluid approximations taking into account mass, momentum, and energy transport along a field line that crosses from the solar photosphere, through the chromosphere and corona, and into the heliosphere. We tested a number of models, and found that none is currently consistent with the observational data for solar wind from coronal holes and from streamers.

There are a number of limitations to our analysis. We assume that all heavy ions propagate at the speed of the solar wind. This may be a good assumption for slower wind, which is generally observed to be closer to a thermal configuration. But solar wind from coronal holes may not be consistent with this assumption (Marsch 1998). We also assume that electron distributions are Maxwellian. However, the presence of nonthermal high-energy tails of electrons in the velocity distribution might alter significantly the ionization and recombination rates, and thus affect the predicted evolution of ion charge states (Owocki and Scudder 1983). While relatively common in flares, the presence of such tails in quiescent solar plasmas is unclear.

However, Feldman et al. (2007) were able to place an upper limit of $5 \%, 2.5 \%$, and $0 \%$ to the percentage of nonthermal electrons with energies of $0.91,1.34$, and $1.85 \mathrm{keV}$, respectively, in order to reproduce spectral lines emitted by active region plasmas. Ralchenko et al. (2007) found that quiet Sun spectral lines were compatible with a two Maxwellian temperature distribution, provided that no more than $5 \%, 3 \%$, and $1 \%$ belonged to a second Maxwellian distribution with peak energy of 300-400, 500, and $1000 \mathrm{eV}$.

Within the limitations of our analysis, we find that models tend to underestimate the ionic charge states of heavy ions. This is not actually caused by the fact that the coronal electron temperatures in the model do not reach the necessary freeze-in temperatures. However, especially for ions freezing in early in the corona, the heavies move too fast through the temperature maximum and do not have time to adjust. A slower solar wind model should create higher freeze-in temperatures. The same effect can be achieved if the solar wind is actually not emitted in steady state from the corona but released unsteadily out of a heated structure, like in the loop reconnection scenario suggested by Fisk (2003).

Acknowledgements This work was supported, in part, by NASA contracts NNX10AQ61G, NNX08AI11G, and NNH08ZDA001N-HGI. T.H.Z. acknowledges the hospitality of the team of the International Space Science Institute, where most of his work on this paper was completed. L.Z. also acknowledges support from NASA's Living With a Star Heliophysics Postdoctoral Fellowship Program, administered by the University Corporation for Atmospheric Research (NCAR), which is supported by the National Science Foundation.

Open Access This article is distributed under the terms of the Creative Commons Attribution License which permits any use, distribution, and reproduction in any medium, provided the original author(s) and the source are credited.

\section{References}

S.K. Antiochos, Z. Mikić, V.S. Titov, R. Lionello, J.A. Linker, A model for the sources of the slow solar wind. Astrophys. J. 731 (2011a). doi:10.1088/0004-637X/731/2/112 
S.K. Antiochos, J.A. Linker, R. Lionello, Z. Mikić, V. Titov, T.H. Zurbuchen, The structure and dynamics of the corona-heliosphere connection. Space Sci. Rev. (2011b). doi:10.1007/s11214-011-9795-7

G. Borrini, J.M. Wilcox, J.T. Gosling, S.J. Bame, W.C. Feldman, Solar wind helium and hydrogen structure near the heliospheric current sheet—a signal of coronal streamers at 1 AU. J. Geophys. Res. 86, 45654573 (1981)

Y. Chen, X. Li, H.Q. Song, Q.Q. Shi, S.W. Feng, L.D. Xia, Intrinsic instability of coronal streamers. Astrophys. J. 691 (2009). doi:10.1088/0004-637X/691/2/1936

S.R. Cranmer, A.A. van Ballegooijen, R.J. Edgar, Self-consistent coronal heating and solar wind acceleration from anisotropic magnetohydrodynamic turbulence. Astrophys. J. 171 (2007). doi:10.1086/518001

J.-P. Delaboudinière, G.E. Artzner, J. Brunaud, A.H. Gabriel, J.F. Hochedez, F. Millier, X.Y. Song, B. Au, K.P. Dere, R.A. Howard, R. Kreplin, D.J. Michels, J.D. Moses, J.M. Defise, C. Jamar, P. Rochus, J.P. Chauvineau, J.P. Marioge, R.C. Catura, J.R. Lemen, L. Shing, R.A. Stern, J.B. Gurman, W.M. Neupert, A. Maucherat, F. Clette, P. Cugnon, E.J. Van Dessel, EIT: Extreme-ultraviolet imaging telescope for the SOHO mission. Sol. Phys. 162, 291-312 (1995)

V. Domingo, B. Fleck, A.I. Poland, The SOHO mission: an overview. Sol. Phys. 162, 1-37 (1995)

R. Esser, R.J. Edgar, Differential flow speeds of ions of the same element: effects on solar wind ionization fractions. Astrophys. J. 563 (2001). doi:10.1086/323987

U. Feldman, K.G. Widing, Elemental abundances in the solar upper atmosphere derived by spectroscopic means. Space Sci. Rev. 107, 665-720 (1999)

U. Feldman, K.G. Widing, H.P. Warren, Morphology of the quiet solar upper atmosphere in the $4 \times 10^{4}<$ $T_{e}<1.4 \times 10^{6} \mathrm{~K}$ temperature regime. Astrophys. J. 522, 1133-1147 (1999)

U. Feldman, E. Landi, G.A. Doschek, Diagnostics of suprathermal electrons in active-region plasmas using He-like UV lines. Astrophys. J. 660 (2007). doi:10.1086/513729

L.A. Fisk, Acceleration of the solar wind as a result of reconnection of open magnetic flux with coronal loops. J. Geophys. Res. 108 (2003). doi:10.1029/2002JA009284

L.A. Fisk, T.H. Zurbuchen, Distribution and properties of open magnetic flux outside of coronal holes. J. Geophys. Res. 111 (2006). doi:10.1029/2005JA011575

J. Geiss, G. Gloeckler, R. von Steiger, H. Balsiger, L.A. Fisk, A.B. Galvin, F.M. Ipavich, S. Livi, J.F. McKenzie, K.W. Ogilvie, B. Wilken, The southern high-speed stream: results from the SWICS instrument on Ulysses. Science 268, 1033-1036 (1995)

G. Gloeckler, J. Geiss, H. Balsiger, P. Bedini, J.C. Cain, J. Fischer, L.A. Fisk, A.B. Galvin, F. Gliem, D.C. Hamilton, J.V. Hollweg, F.M. Ipavich, R. Joss, S. Livi, R. Lundgren, U. Mall, J.F. McKenzie, K.W. Ogilvie, F. Ottens, W. Rieck, E.O. Tums, R. von Steiger, W. Weiss, B. Wilken, The solar wind ion composition spectrometer. Astron. Astrophys. Suppl. Ser. 92(2), 267-289 (1992)

G. Gloeckler, T.H. Zurbuchen, J. Geiss, Implications of the observed anticorrelation between solar wind speed and coronal electron temperature. J. Geophys. Res. 108 (2003). doi:10.1029/2002JA009286

J. Gosling, Physical nature of low-speed solar wind, in Robotic Exploration Close to the Sun, ed. by S.R. Habbal, AIP Conf. Proc., vol. 385 (AIP, Woodbury, 1997), pp. 17-24

J.R. Gruesbeck, S.T. Lepri, T.H. Zurbuchen, S.K. Antiochos, Constraints on coronal mass ejection evolution from in situ observations of ionic charge states. Astrophys. J. 730 (2011). doi:10.1088/ 0004-637X/730/2/103

M. Hahn, E. Landi, D.W. Savin, Differential emission measure analysis of a polar coronal hole during the solar minimum in 2007. Astrophys. J. 736, 101 (2011)

V.H. Hansteen, E. Leer, Coronal heating, densities and temperatures and solar wind acceleration. J. Geophys. Res. 100, 21577-21593 (1995)

Y.-K. Ko, L.A. Fisk, J. Geiss, G. Gloeckler, M. Guhathakurta, An empirical study of the electron temperature and heavy ion velocities in the south polar coronal hole. Sol. Phys. 171, 345-361 (1997)

J.L. Kohl, G. Noci, E. Antonucci, G. Tondello, M.C.E. Huber, S.R. Cranmer, L. Strachan, A.V. Panasyuk, L.D. Gardner, M. Romoli, S. Fineschi, D. Dobrzycka, J.C. Raymond, P. Nicolosi, O.H.W. Siegmund, D. Spadaro, C. Benna, A. Ciaravella, S. Giordano, S.R. Habbal, M. Karovska, X. Li, R. Martin, J.G. Michels, A. Modigliani, G. Naletto, R.H. O’Neal, C. Pernechele, G. Poletto, P.L. Smith, R.M. Suleiman, UVCS/SOHO empirical determinations of anisotropic velocity distributions in the solar corona. Astrophys. J. 501, L127-L131 (1998)

J.M. Laming, S.T. Lepri, Ion charge states in the fast solar wind: new data analysis and theoretical refinements. Astrophys. J. 660 (2007). doi:10.1086/513505

E. Landi, R.L. Alexander, J.R. Gruesbeck, J.A. Gilbert, S.T. Lepri, W.B. Manchester, T.H. Zurbuchen, Carbon ionization stages as a diagnostic of the solar wind. Astrophys. J. 744, 100-112 (2012)

E. Leer, V.H. Hansteen, E. Holzer, Understanding the solar wind, in Cyclical Variability in Stellar Winds, ed. by L. Kaper, A.W. Fullerton. Proceedings of the ESO Workshop (Springer, Berlin, 1998), p. 263

S.T. Lepri, T.H. Zurbuchen, L.A. Fisk, I.G. Richardson, H.V. Cane, G. Gloeckler, Iron charge distribution as an identifier of interplanetary coronal mass ejections. J. Geophys. Res. 106, 29,231-29,238 (2001) 
E. Marsch, Closure of multi-fluid and kinetic equations for cyclotron-resonant interactions of solar wind ions with Alfvén waves. Nonlinear Process. Geophys. 5, 111-120 (1998)

P. Mazzotta, G. Mazzitelli, S. Colafrancesco, N. Vottorio, Ionization balance for optically thin plasmas: rate coefficients for all atoms and ions of the elements H to Ni. Astron. Astrophys. 133, 403-409 (1998)

S.P. Owocki, J.D. Scudder, The effect of a non-Maxwellian electron distribution on oxygen and iron ionization balances in the solar corona. Astrophys. J. 270, 758-768 (1983)

Y. Ralchenko, U. Feldman, G.A. Doschek, Is there a high-energy particle population in the quiet solar corona? Astrophys. J. 659 (2007). doi:10.1086/512536

N.A. Schwadron, D.J. McComas, Solar wind scaling law. Astrophys. J. 599 (2003). doi:10.1086/379541

R. Schwenn, Large-scale structure of the interplanetary medium, in Physics of the Inner Heliosphere I. Space and Solar Physics, vol. 20 (Springer, Berlin, 1990), pp. 99-181

R. Schwenn, Solar wind sources and their variations over the solar cycle, in Solar Dynamics and Its Effects on the Heliosphere and Earth. Space Sciences Series of ISSI, vol. 22 (2007), pp. 51-76

N.R. Sheeley, Y.-M. Wang, S.H. Hawley, G.E. Brueckner, K.P. Dere, R.A. Howard, M.J. Koomen, C.M. Korendye, D.J. Michels, S.E. Paswaters, D.G. Socker, O.C. St. Cyr, D. Wang, P.L. Lamy, A. Liebaria, R. Schwenn, G.M. Simnett, S. Plunkett, D.A. Blesecker, Measurements of flow speeds in the corona between 2 and 30 R $\odot$. Astrophys. J. 484 (1997). doi:10.1086/304338

B. van der Holst, W.B. Manchester, R.A. Frazin, A.M. Vásquez, G. Tóth, T.I. Gombosi, A data-driven, twotemperature solar wind model with Alfvén waves. Astrophys. J. 725 (2010). doi:10.1088/0004-637X/ $725 / 1 / 1373$

R. von Steiger, The solar wind through the solar cycle, in The Heliosphere through the Solar Activity Cycle, ed. by A. Balogh, L.J. Lanzerotti, S.T. Suess (Springer Praxis, London, 2008), pp. 41-78

R. von Steiger, T.H. Zurbuchen, Kinetic properties of heavy solar wind ions from Ulysses-SWICS. Geophys. Res. Letts. 33 (2006). doi:10.1029/2005GL024998

R. von Steiger, T.H. Zurbuchen, Polar coronal holes during the past solar cycle: Ulysses observations. J. Geophys. Res. 116 (2011). doi:10.1029/2010JA015835

R. von Steiger, N.A. Schwadron, L.A. Fisk, J. Geiss, G. Gloeckler, S. Hefti, B. Wilken, R.R. WimmerSchweingruber, T.H. Zurbuchen, Composition of quasi-stationary solar wind flows from Ulysses/solar wind ion composition spectrometer. J. Geophys. Res. 105(A12), 27,217-27,238 (2000)

R. von Steiger, T.H. Zurbuchen, D.J. McComas, Oxygen flux in the solar wind: Ulysses observations. Geophys. Res. Letts. 37(22) (2010). doi:10.1029/2010GL045389

Y.-M. Wang, N.R. Sheeley Jr., J.P. Phillips, B.E. Goldstein, Solar wind stream interactions and the wind speed expansion factor relationship. Astrophys. J. 488, L51-L54 (1997)

K.-P. Wenzel, R.G. Marsden, D.E. Page, E.J. Smith, The Ulysses mission. Astron. Astrophys. Suppl. Ser. 92, 207-219 (1992)

R.F. Wimmer-Schweingruber, R. von Steiger, R. Paerli, Solar wind stream interfaces in corotating interaction regions: SWICS/Ulysses results. J. Geophys. Res. 102(A8), 17,407-17,418 (1997)

L. Zhao, L.A. Fisk, Understanding the behavior of the heliospheric magnetic field and the solar wind during the unusual solar minimum between cycles 23 and 24. Solar Phys. 274(1-2), 379-397 (2011). doi:10.1007/s11207-011-9840-4

L. Zhao, T.H. Zurbuchen, L.A. Fisk, Global distribution of the solar wind during solar cycle 23: ACE observations. Geophys. Res. Letts. 36 (2009). doi:10.1029/2009GL039181

T.H. Zurbuchen, A new view of the coupling of the Sun and the heliosphere. Annu. Rev. Astron. Astrophys. 45, 297-338 (2007)

T.H. Zurbuchen, I.G. Richardson, In-situ solar wind and magnetic field signatures of interplanetary coronal mass ejections, in Coronal Mass Ejections. Space Science Series of ISSI, vol. 21 (2006), pp. 31-43

T.H. Zurbuchen, R. von Steiger, On the solar wind elemental composition: constraints on the origin of the solar wind, in 10 Years of SOHO and Beyond, ed. by H. Lacoste, L. Ouwehand. ESA SP-617 (ESA, Paris, 2006), p. 7.1

T.H. Zurbuchen, L.A. Fisk, G. Gloeckler, R. von Steiger, The solar wind composition throughout the solar cycle: a continuum of dynamic states. Geophys. Res. Letts. 29(9) (2002). doi:10.1029/2001GL013946 